\title{
Risposte esatte ai test di verifica apparsi su Tecniche Nefrologiche \& Dialitiche $\mathbf{N}^{\circ} 2$
}

\section{La peritonite sclerosante in breve}

\section{G. d'Adamo}

\section{PAG 18}

\section{TEST DI VERIFICA - 1}

1) La peritonite sclerosante è più frequente

a) nei pazienti anziani?

b) nei pazienti in dialisi peritoneale da molti anni?

c) nei diabetici?

d) nelle donne?

2) La peritonite sclerosante può comparire
a) nei primi anni di dialisi peritoneale?
b) dopo il passaggio in emodialisi?
c) dopo il trapianto?
d) dopo un episodio di peritonite?
e) in tutti questi casi

risposta esatta

\section{Approccio diagnostico al sanguinamento gastrointestinale occulto nel paziente in trattamento emodialitico}

\section{U. Brummer, P. Cappelli, L. Amoroso, L. Di Liberato, P. Muscianese,}

V. Sirolli, A. Perilli, M. Neri, M. Bonomini

PAG 24

TEST DI VERIFICA - 2

1) Quale è la localizzazione più frequente dell'angiodisplasia gastrointestinale nel paziente in dialisi:
a) colon
b) retto
c) stomaco e duodeno
d) digiuno

risposta esatta

2) Quale di queste affermazioni sull'angiodisplasia è vera:
a) il sanguinamento è spesso autolimitan- tesi
b) il sanguinamento è spesso cronico
c) il sanguinamento può essere ricorrente
d) tutte le precedenti 


\title{
Insufficienza renale acuta in un paziente con sarcoidosi renale isolata e carcinoma uroteliale occulto bilaterale
}

\author{
M. Quaglia, M. Salomone, R. Fenoglio, E. Lazzarich, C. Musetti, L. Gravellone, \\ E. Karvea, C. Bozzola, P. Stratta
}

PAG 29

TEST DI VERIFICA - 3

1) Quali di questi contesti clinici, oltre alla sarcoidosi, si associa a nefrite interstiziale granulomatosa?
a) infezione da toxoplasma
b) Infezione da HIV
risposta esatta
c) Terapia con diuretici a forti dosi
d) Terapia con Cisplatino

2) Quali di queste cause di insufficienza renale acuta, oltre a quella descritta di nefrite interstiziale granulomatosa, può verificarsi in corso di sarcoidosi?

a) danno tubulotossico diretto da eccesso di ACE

b) meccanismo prerenale secondario agli effetti della ipercalcemia

c) necrosi papillare

d) sindrome emolitico-uremica

\section{Difficoltà diagnostiche nella glomerulonefrite post-infettiva: un caso clinico}

\section{Rossi, C. Savignani, G. Solano, L. Pittavini, R. Brugnano, C. Covarelli, A. Sidoni}

\section{PAG 47}

\section{TEST DI VERIFICA - 4}

1) Glomerulonefrite post-infettiva (GNPI) è sinonimo di glomerulonefrite post-streptococcica?
a) sì

b) no

2) Il quadro clinico di esordio di più frequente riscontro nella GNPI batteriche è:

a) proteinuria $>3 \mathrm{gr} / 24$ ore, edemi, insufficienza renale

b) sindrome nefrosica

c) ematuria , proteinuria , edemi, ipertensione arteriosa, ipocomplementemia.
Commento alla risposta esatta 1 = le glomerulonefriti post-infettive comprendono una varietà di quadri clinico-patologici da agenti causali batterici, virali e parassitari. La GNF post-streptococcica è la forma più diffusa con una incidenza tra il 6 ed il 10\% delle GN istologicamente definite ed è in riduzione nell'adulto nei Paesi occidentali. L'agente eziologico in causa è lo streptococco emolitico gruppo A con i sierotipi 1,2,4,12,18,25,49,55,57,60

risposta esatta

commento alla risposta esatta 2 = Le GNPI batteriche si manifestano più frequentemente con un quadro di sindrome nefritica con ematuria ( macro e microscopica di origine glomerulare), cilindruria, proteinuria che può risultare in range nefrosico, edemi, ipertensione arteriosa, oligo-anuria. Le alterazioni di laboratorio più frequenti sono una transitoria riduzione del complemento, la presenza di immunocomplessi circolanti e crioglobuline, un elevato livello di $\operatorname{IgG}$ sieriche, un aumento degli indici di flogosi. I quadri clinici più severi si osservano in soggetti immunocompromessi (diabetici, alcoorisposta esatta listi, neoplastici, tossicodipendenti) . 


\section{Un caso di ipertensione arteriosa con rene piccolo}

\section{A. Mannarino, P. Becherelli \\ PAG 50, 51 \\ TEST DI VERFICA - 5}

1) Qual è l’ipotesi diagnostica più verosimile?

a) ipoplasia renale semplice

b) nefropatia da reflusso con rene $\mathrm{dx}$ pielenefritico

c) fibrodisplasia arteria renale $d x$

d) fibrodisplasia bilaterale arteria renale

e) stenosi arteriosa aterosclerotica unilaterale

\section{TEST DI VERIFICA - 6}

1) Quali sono le possibili cause di ipertensione?

a) nefropatia parenchimale cronica

b) ipertensione arteriosa nefrovascolare 1 kidney - 1 clip risposta esatta

c) ipertensione arteriosa nefrovascolare 2 kidney - 1 clip

d) ipertensione arteriosa essenziale

e) altre cause secondarie di ipertensione

2) Quali indagini avresti eseguito?
a) angio ct multistrato risposta esatta
b) scintigrafia renale sequenziale basale e dopo ACEi
c) arteriografia selettiva arteriosa renali
d) scintigrafia renale con DMSA
e) cistografia minzionaale 


\section{Un caso particolare "molto generale"}

\section{S. Geatti, L. Feltrin}

\section{PAG 55}

\section{TEST DI VERIFICA - 7}

1 In relazione al contenuto del box n. 1, l'approccio dell'infermiere rispetto alle indicazioni alimentari del paziente dializzato si è rivelato:

a) Efficace: contenuti adeguati e modalità comunicative adatte alla situazione

b) Non efficace: inadeguato nei contenuti e nelle delle modalità comunicative

c) Parzialmente efficace: tratti accettabili ma non completi nei contenuti e nelle modalità comunicative risposta esatta

2 Quali strategie potevano essere adottate per una più efficace azione educativa

a) Contatto con la badante

b) Attivazione dell'assistenza domiciliare

c) Accertamento infermieristico sul caso più appropriato risposta esatta

3 In un piano educativo alimentare affrontando il problema dell'iperpotassiemia, indicare l'ordine di priorità delle seguenti azioni (1-3)

2) Illustrare le relazioni tra singoli alimenti e contenuto di potassio

3) Definire un modello alimentare equilibrato rispetto alle caratteristiche del paziente

1) Illustrare al paziente i rischi dell'iperpotassiemia e renderlo capace di riconoscerne la sintomatologia

risposta esatta 2-3-1

4 Considerando il caso affrontato, l'intervento educativo dell'infermiera:

a) E stato adeguato, perché ha stimolato le capacità residue del paziente e perché ha considerato le risorse di supporto

b) E stato in parte inadeguato perché ha fornito strumenti ma non ne ha approfondito sufficientemente le modalità per realizzarli risposta esatta

c) E stato inadeguato perché non indaga a sufficienza sulle capacità cognitive e di gestione della terapia del paziente e non sottolinea l'importanza di una corretta assunzione degli antipertensivi 


\title{
Il rigetto acuto umorale nel trapianto renale:
}

\section{caratteristiche cliniche, diagnosi e trattamento}

\author{
A. Rosati, E. Bertoni, L. Di Maria, A. Larti, G. Rosso, M. Salvadori
}

PAG 63

\section{TEST DI VERIFICA - 8}

1) Quale delle seguenti affermazioni, a proposito del rigetto umorale, è corretta?
a) La plasmaferesi è indicata solo quando il livello di anticorpi è $>20 \%$
b) La plasmaferesi non deve essere mai utilizzata nel primo mese post-trapianto
c) La plasmaferesi può essere sostituita dall'immunoassorbimento risposta esatta
d) La plasmaferesi è controindicata in caso di retrapianto

2) Quali sono i farmaci più frequentemente utilizzati per il trattamento del rigetto umorale?
a) Thimoglobuline
b) Sirolimus e Steroide
c) Plasmaferesi, immunoglobuline ad alte dosi
d) Tacrolimus e MMF
e) $a+b$
f) $\mathrm{a}+\mathrm{c}+\mathrm{d}$
g) Nessuna delle precedenti

risposta esatta

3)La proteinuria nel trapianto è un fattore di rischio indipendente di perdita dell'organo quando:

a) E superiore a $250 \mathrm{mg} / 24 \mathrm{~h}$

b) È superiore a $500 \mathrm{mg} / 24 \mathrm{~h}$ risposta esatta

c) È superiore a $1 \mathrm{~g} / 24 \mathrm{~h}$

d) $\mathrm{E}$ superiore a $2 \mathrm{~g} / 24 \mathrm{~h}$

e) Non è un fattore di rischio di perdita dell'organo 